\title{
Mobility, Remobilisation, Exercise and Prevention of the Complications of Stasis
}

\author{
Panagiota Copanitsanou
}

The positive effects of physical activity on physical and mental health are well known and include weight control, improved balance, flexibility, strength, anxiety reduction and protection from ill health, as well as contributing to independent living and preventing falls. Being mobile and able to self-care and fear of falling are important to patients. A central goal of nursing care following fragility fracture is to maximise mobility. Individual patient goals are determined by their pre-fracture mobility and functional status. Recovery is often compromised for those with limited pre-fracture activity and cognitive impairment, low functional levels postoperatively, older age, polypharmacy, comorbidities, depression, poor nutritional status, lack of social support and not living independently. Many patients never recover their previous level of function after a fragility fracture and there is significant risk of institutionalisation, new fractures, disability and loss of independence.

Patients should undergo multidisciplinary assessment to identify factors known to be associated with risk for poor functional recovery so that appropriate multidisciplinary interventions can be implemented. The aim of this chapter is to highlight the risks of immobility and the benefits of remobilisation and exercise to enable clinicians to effectively manage the multiple and interconnected individual factors of each patient to maximise their function.

\footnotetext{
P. Copanitsanou

Department of Orthopaedic and Traumatology, General Hospital of Piraeus "Tzaneio", Piraeus, Greece

National and Kapodistrian University of Athens, Athens, Greece

e-mail: gcopanitsan@nurs.uoa.gr 


\subsection{Learning Outcomes}

At the end of the chapter, and following further study, the nurse will be able to:

- Identify the risks of immobilisation and benefits of remobilisation and exercise

- Highlight the importance of mobilisation and exercise in the prevention of complications and effective recovery

- Recognise the factors that affect mobility

- Define which factors must be considered when assessing patients' mobility and capacity for remobilisation and exercise

- Describe the most effective approach for pain assessment and management

- Define the relationship between immobility and stasis and complications following hip fracture

- Outline the evidence-based measures for the prevention of venous thromboembolism, urinary tract infection, constipation and pneumonia

- Implement appropriate exercises and mobilisation strategies and document care

- Confidently support older people with fragility fractures in their difficult journey to recovery.

\subsection{Mobility and Remobilisation}

Mobilisation is essential for health-related quality of life and independence. For older people following hip fracture, early mobilisation is especially important because it is linked to mortality [1] and functional recovery [2] as well as risk of functional decline due to the injury, perioperative immobilisation, muscle weakness, fatigue and postoperative complications.

Pain limits remobilisation and is associated with delirium, depression, sleep disturbances and poor mobility. Muscle strength deficit in the fractured limb is associated with even greater pain and it is unethical to expect patients to comply with rehabilitation exercises without managing pain effectively. Good pain management helps avoid delays in rehabilitation, postoperative complications, delayed discharge and unsafe mobility.

Patients also suffer from loss of confidence, fear of falling and are at risk of further fractures and other complications. Older women have been found to prefer being dead than experience loss of independence [3], demonstrating the psychological impact. Those who are not remobilised early may feel demoralised, so it is important that they have realistic expectations to avoid disappointment. Management of the fear of falling is also central, along with the need to educate patients and carers about fall prevention and the importance of exercise.

There are numerous factors that affect ability to remobilise. The progressive loss of muscle mass with ageing is associated with decreasing reserves and sarcopenia (Chap. 2) as well as immobility. For every day spent in bed, 2.5 days are needed to regain the strength to walk [4]. Frailty leads to poor outcomes and affects capacity for mobilisation and exercise. Other conditions to consider include depression, 
cognitive impairment and delirium (Chap. 9), which are also associated with poorer mobility outcomes and limit participation in exercise. The main factors that impact on ability to remobilise are summarised in Box 6.1.

It is essential to consider the impact of these multiple factors on exercise and mobility and take an individualised, holistic approach. Patients who were mobile before the fracture should be mobilised regardless of their cognitive status and the focus should be on gait quality, walking endurance, activities of daily living and safety [5].

\section{Box 6.1: Factors Affecting Ability to Mobilise After Fragility Fracture}

- Pain

- Loss of confidence, fear of falling, fear of refracturing the bone, fear of other local/ surgical complications

- Osteoporosis

- Comorbidities, multimorbidity

- Polypharmacy

- Nutritional status

- Progressive muscle loss, sarcopenia

- Frailty

- Cognitive impairment, depression, delirium

\subsection{Exercise}

Mobilisation strategy, type of weight bearing, timing and progress of exercise depend on the type of fracture and surgery and there are contradictions about evidence-based pathways for management after different fractures and procedures; e.g. after hemiarthroplasty, mobilisation may start earlier, while following extracapsular fracture, it may be delayed. Surgeons usually decide when to allow restricted or full weight bearing [6], with a mean time for an order for ambulation of 2 days, as some hesitate because of concerns about mechanical failure [7]. However, delay in weight bearing has been connected to poor function [8]. With current implants and surgical techniques, most patients can be allowed to weight bear and movements (e.g. crossing the legs past the midline of the body, avoiding bending or overreaching) should not be restricted. Familiarity with basic exercises (i.e. foot and ankle, static quadricep/gluteal/abdominal, knee extension/flexion, hip abduction) as well as functional exercises is essential. However, walking may be compromised by practical obstacles such as wound drains, intravenous infusion devices and the surgical wound.

Nurses should encourage patients to sit in a chair for meals as soon as possible and encourage them to be independent in self-care and hygiene. All staff should be involved in encouraging independence in toileting and transfers and in making daily assessments of patients' progress so that they can determine individual needs and prevent delays in transfers and discharge. 
Effective pain management is central in enabling patients to exercise, sleep well and promote recovery. A baseline pain assessment (pain history, previous use of pain medications) should be performed, and mobilisation and pain management should be coordinated (i.e. correct timing of medication administration in relation to exercise sessions). Patients' self-report is the gold standard for assessing pain. Assessment should be conducted using numerical, verbal, facial, or visual analogue scales. Adjustments in medication dose may be needed based on individual responses, as some patients may become sedated, while others may need higher doses. Pain management interventions should not only be pharmacological but also include non-pharmacological options such as transcutaneous electrical nerve stimulation, distraction, muscle relaxation, acupressure, heat/ice and relaxation techniques. Multiple strategies should be used in combination. A self-reported reduction of pain by $20-30 \%$ is considered effective [9].

Patients do not always receive adequate pain management, especially those with dementia and/or delirium who have more difficulty reporting pain [10], and behavioural (e.g. moans, sighs, restlessness, agitation, rapid blinking, facial expressions) or physiological (e.g. tachycardia, high blood pressure) signs are rarely considered. Effective pain assessment requires familiarity with the patient and information from carers [11]. Pain may not only be acute (up to 30 days postfracture/surgery) but may also be chronic [9]. Although some discomfort is expected during the first few months, patients must be able to differentiate between discomfort and pain [4]. Nurses should inform patients about when increased pain indicates a problem and about the avoidance of exercise when strain on the surgical area is experienced.

To minimise the risk for falling (Chap. 3) and for remobilisation to be safe, patients should be actively involved in their own care. Assistance should be provided as needed for them to remain as functional and active as possible. Even if they cannot perform exercises alone, they must not remain immobilised but should undertake simple exercises in bed or while sitting in a chair. Nurses need to know how to help patients to mobilise safely as in-hospital falls are a nursing care quality indicator.

Patient education should include the type of pain medications and time intervals for administration, the importance of receiving medications before the pain becomes intense, coordination between the administration of pain medication and exercise and the interactions of medications. Patients should be reminded that exercise helps to reduce pain and that pain improves more quickly if they stay active and achieve a balance between activity and rest [4].

\subsection{The Complications of Stasis}

Nearly half of patients with a hip fracture develop at least one complication [12]. Surgical management enables earlier mobilisation and prevents complications of prolonged immobilisation (e.g., urinary tract infections, pressure ulcers, respiratory/cardiac/renal/gastrointestinal complications, venous thromboembolism). 


\subsubsection{Venous Thromboembolism}

Deep vein thrombosis (DVT) following hip fracture has a frequency of $1-24 \%$, depending on the screening method used, while the frequency of fatal pulmonary embolism ranges between 0.5 and $-7.5 \%$ [10]. Patients with fragility fracture are susceptible to venous thromboembolism (VTE) due to ageing, the fracture itself, immobilisation, hospitalisation and surgery. Other risk factors include history of previous thromboembolism, malignancy, congestive heart failure, obesity and vascular disease.

\subsubsection{Pulmonary and Urinary Tract Infection}

Hospital-acquired infections cause significant morbidity and must be prevented. Postoperatively, pulmonary complications are among the most frequent and their occurrence can increase from $6.3 \%$ preoperatively to $10.7 \%$ postoperatively [13]. Complications such as atelectasis and pneumonia contribute to increased length of hospital stay and mortality [14]. The risk factors for the development of a pulmonary infection include chronic respiratory disease, male gender, use of steroids, number of comorbidities and older age [15].

The frequency of urinary tract infections (UTI) has been reported to be as low as $2 \%$ [13] and as high as 52\% [16]. UTI is associated with increased length of hospital stay and poor functional outcomes and is usually caused by the use of indwelling catheters. Urinary catheters also cause restriction of mobility, pain, delirium and increased mortality [17].

Indwelling urinary catheters are often inserted on admission or are used postoperatively to accommodate the patients' limited independence. However, the reason for inserting an indwelling catheter is often unclear [18] and should be specifically recorded, e.g. urinary retention unrelieved by intermittent catheterisation, skin problems in the perineal area/sacrum area, for close monitoring of cardiac or renal function or as a comfort measure in end of life care [17]. Patients with indwelling catheters are more likely to have positive urine cultures compared to following intermittent catheterisation [18] and the risk of UTI increases an estimated 5-10\% for every $48 \mathrm{~h}$ of indwelling urinary catheter placement [19]. Those most at risk are older patients, women [20], those with dehydration and/or poor nutrition postoperatively [21] and those with diabetes or malignancy [13].

\subsubsection{Constipation}

Constipation often occurs postoperatively because of analgesics, limited activity and lack of privacy but is a commonly overlooked aspect of care; $69 \%$ of patients with fragility fractures develop constipation during the first postoperative days and $62 \%$ at 30 days after surgery. In $22.7 \%$ of patients, a normal defecation pattern may not be re-established even within 30 days [22]. Risk factors include dehydration, 
immobility, decreased dietary fibre, changes to normal dietary routines and opioid analgesics (even in low doses). Opioid-induced constipation may cause patients to refuse pain medications, thus compromising pain relief and remobilisation. Patients may not inform nurses of their constipation symptoms and nurses may not initiate a conversation. The presence of constipation, symptoms, use of laxatives and impact on pain management is often not fully appreciated by healthcare professionals.

\subsection{Assessment of Mobility and Remobilisation Potential}

Mobility status affects patient handling and outcomes, so mobility, remobilisation and exercise ability should be carefully assessed taking into account patients' function, cognitive and psychosocial status. Due to their 24-hour presence and ability to perform continuous assessment of progress, nurses do not need to rely on other healthcare professionals to determine patients' mobility. The criteria for assessing mobility are subjective because quantifiable terms for mobility are rarely used. A summary of factors to consider when assessing mobility is provided in Box 6.2.

Assessing mobility using tests for physical function (e.g. range of motion) is limited, as tests may be unreliable between different observers. Likewise, the observation of mobilisation can be intrusive or may only convey "snapshots" of patients' abilities unless observation takes place on several occasions over a period of time. By interviewing patients, information can be gathered about their views regarding their situation, strengths and problems, but even this may be compromised by difficulties in communication and by patients' cognitive impairment as well as their desire to appear better than they are [23].

Several tools have been developed for assessment of mobility and remobilisation ability. Options include the New Mobility Score, the Quick 5 Bedside Guide tool, the Hand Grip measurement, the Berg Balance Scale, the de Morton Mobility Index, the Modified Elderly Mobility Scale, the Timed Up and Go Test, the Banner Mobility Assessment Tool, the Tinetti Assessment Tool, the Barthel Index, the Egress Test, the Functional Independence Measure, the Functional Assessment Measure, the Performance-Oriented Mobility Assessment and the Elderly Mobility Scale. Although these tools are used in hospitals, they may be inappropriate or of limited value for nurses in acute-care settings. There is a need to understand how they should be used and their validity, reliability and practicability, as well as any copyright restrictions. The information gathered can help to inform history taking and can be used to compare and document progress throughout rehabilitation [23].

The assessment of mobility potential has not only to do with mobility itself but includes individual goals, safety and use of appropriate walking aids. Safety is an important parameter and nurses need to know how to assess individual risks, how to manage them, how to use appropriate aids, how to safely handle each patient and how to ensure the environment is safe.

Other, more targeted, assessments may include that of the musculoskeletal system (e.g. muscle mass/strength, sarcopenia, presence of arthritis, osteoporosis, neuromusculoskeletal disorders), pre-fracture mobility and lifestyle (e.g. dependent, 
sedentary, independent), cognitive and psychosocial status, any visual and/or hearing disorders, the presence of significant others, the patient's own beliefs and willingness for mobilisation and their educational needs regarding mobility/remobilisation.

Box 6.2: Assessment of Mobility and Remobilisation Potential

- General physical health status

- Assessment of musculoskeletal system (e.g. muscle mass/strength, sarcopenia, presence of arthritis, osteoporosis, neuro-musculoskeletal disorders)

- Pain

- Lifestyle before the fracture (dependent, sedentary, independent)

- Cognitive status, depression, delirium

- Visual and/or hearing disorders

- Presence of family/significant others

- Patient's beliefs and willingness for remobilisation

- Educational needs regarding mobility/remobilisation

\subsection{Evidence-Based Interventions for Mobility and Remobilisation}

Rehabilitation should start as soon as possible postoperatively once the patient is medically stable. There is a series of actions that nurses need to perform both as members of the multidisciplinary team (MDT) and independently to promote this (a summary is provided in Box 6.3). Through patient-centred participation and collaboration with the MDT and with the patient participating in the goal-setting, nurses help to set individual and realistic goals and plan interventions that support individual preferences and actively encourage patients in self-care and independence. When a patient is transferred or discharged, nurses should communicate any successful strategies or risks observed during hospitalisation to other healthcare professionals [5].

Nurses play a key role in the patients' psychological and biophysiological preparation for remobilisation and they can provide sustained encouragement. Through therapeutic communication with patients, nurses can ensure they are treated as individuals and that they are supported after their great trauma towards a future of independent life and to believe in the possibility that this is achievable. In acute trauma wards, there is often limited opportunity for physical activity [24], but nurses can encourage patients to remain physically active and participate in self-care so that functional decline is reduced [25].

Patient education, as an independent nursing intervention, can be used to inform patients about the importance of mobilisation and exercise, complication prevention, rehabilitation programme and long-term outcomes. Education helps motivate patients to want to move, as they are often reluctant to comply with rehabilitation. Nurses need to be firm in encouraging them to move, but at the same time, they 
should make sure they do so without patronising them. The anxieties of patients and their carers should be recognised and addressed. Family members provide a support network that enhances patient recovery through practical help and psychosocial support and can be a valuable source of information about the patient's pre-fracture status and preferences [24]. High-quality care of older patients relies not only on excellent MDT communication but also on close cooperation with patients and families.

Box 6.3: Evidence-Based Interventions for Mobility and Remobilisation

- Encouraging and supporting in remobilisation, self-care and independence

- Use of safety measures/prevention of falls/walking aids

- Education about exercises (both of patients and family/carers)

- Pain management (before remobilisation)

- Adequate rest/sleep

- Adequate nutrition/hydration

- Participation within the multidisciplinary team (patient-centred)

- Communication of any successful strategies or risks observed during hospitalisation to other healthcare professionals after discharge

\subsection{Exercise: Assessment}

Before the commencement of exercise, there should be an overall assessment of functional level, ability to perform Activities of Daily Living (ADLs) and the assistance needed to accomplish them, sensory ability, cognitive status and capacity to ambulate. This should include an evaluation of gait instability and risk of falls. Older people often view their health in terms of how well they function, not in terms of disease, and it is important to identify strengths as well as need for assistance. Baseline functional status should be documented to facilitate assessment of progress. Assessment should also include information about the type of fracture and surgery (to determine what exercise is feasible or unadvisable) and the needs for walking aids and personal safety measures. For example, the selection of the most appropriate walking stick should be made according to the fracture type as well as hand grip, gait, height and stability.

\subsection{Exercise: Evidence-Based Interventions}

Mobilising patients early leads to reduced length of hospital stay, improved mobility, improved walking distance and overall improved function [26, 27]. Although patients with good pre-fracture level of mobility without cognitive impairment tend to benefit most from rehabilitation, exercise is beneficial even in the presence of functional limitations and/or cognitive impairment $[2,28]$. It is essential to increase muscle strength and range of motion [9], so walking and exercise training aim to minimise 
impairment [6]. The type, frequency and duration of exercise that is recommended postoperatively (sometimes after $\mathrm{x}$-rays to check the stability of fixation and the surgeon's agreement) for each patient are also important factors. Remobilisation should start with simple exercises, while their intensity gradually progress.

There is some evidence that exercise with higher intensity and duration is related to better outcomes [28], while potential risks of intensive exercise appear to be minimal. Specific types of exercises are beneficial such as progressive resistance training and balance training, which can be safe and effective [29]. However, there is insufficient evidence about the best strategies for enhancing mobility and more research is needed to determine the most appropriate type, duration and intensity of exercise, as well as the value of first-day mobilisation.

There are several ways for nurses to help patients comply with exercise programmes and maximise performance. They can encourage patients to maintain a daily routine with physical activity; they can educate them on the physiological and psychological value of independent functioning, assess and treat their pain, ensure a safe environment, emphasise the importance of nutrition and medications and document all interventions and responses.

\subsection{Prevention of Complications of Stasis}

Complications may be caused by limited mobility and, in a vicious circle, they can limit mobilisation potential due to pain, distress and restrictions caused by treatment or safety measures. They can also lead to poorer outcomes and mortality.

\subsubsection{Assessment}

As part of the prevention strategy, patients should be assessed for the presence of signs and symptoms of thromboembolism which can be nonspecific but include pain (especially during dorsiflexion of the foot), tenderness, changes in colour and temperature of the skin, oedema and, in the case of pulmonary embolism; dyspnoea, chest pain, increased respiratory rate and haemoptysis. However, even in the presence of pulmonary embolism, there may be no signs or symptoms, with cardiac arrest being the first manifestation. Medical history and a physical examination should be used to exclude other causes.

Assessment is important in identifying individual risk of pulmonary infections such as being of older age, poor general health, other infections, cardiopulmonary diseases, malnutrition and impaired renal function [14]. Nurses should assess for presence of cough, sputum production, increased respiratory rate, oxygen saturation levels, dyspnoea, elevated temperature, pleuritic pain, rhonchi/wheezes, use of accessory breathing muscles, cyanosis and changes in mental status.

Assessment for UTI should include monitoring for fever, burning during urination/dysuria, urgency and frequency of urination, suprapubic or pelvic pain, haematuria and new onset or worsening of pre-existing confusion/agitation. Urine colour, 
concentration, odour, reduced volume and cloudiness should also be assessed. The presence of asymptomatic bacteriuria, especially in older people, does not necessitate treatment.

Assessment for constipation should include the number of bowel movements per day/week, abdominal distention and discomfort, abdominal or rectal pain, decreased appetite, nausea, vomiting, bowel obstruction, headache, fatigue, agitation and delirium. Nurses should document usual bowel patterns, severity of constipation and any improvements or progression of constipation [17].

\subsubsection{Prevention of Complications}

The prevention of complications enables patients to participate actively in rehabilitation. There is evidence in favour of mobilisation to prevent thromboembolism and urinary tract infections.

\subsubsection{Prevention of Thromboembolism}

There is insufficient evidence for a protocol to be developed regarding thromboembolic prophylaxis following hip fracture. Aspirin significantly reduces DVT and pulmonary embolism (PE) compared to placebo, and, although patients treated with aspirin need more blood transfusions, mortality associated with bleeding is similar. Nevertheless, aspirin is inferior to other methods of prophylaxis. The overall balance of risks and benefits is complex in hip fracture patients [30].

It has been shown that the administration of heparin leads to a reduction in the frequency of lower limb thromboembolism, but not PE, and there seems to be no difference in efficacy between fractionated or unfractionated heparins; low molecular weight heparins have improved bioavailability, have fewer side effects and are easy to use. Treatment usually takes place for 28-35 days. Both low molecular weight heparins and unfractionated heparin should start on admission unless contraindicated, stoped $12 \mathrm{~h}$ preoperatively and restarted 6-12 h postoperatively [30]. Guidelines state that chemoprophylaxis with fondaparinux should continue for 4 weeks after surgery. Fondaparinux seems to be more efficient than low molecular weight heparins in preventing thromboembolism without significant differences in mortality or bleeding. However, it is not recommended for preoperative use in patients with hip fractures; if it is used preoperatively, it should be stopped $24 \mathrm{~h}$ before surgery and restarted $6 \mathrm{~h}$ postoperatively [30].

Mechanical prophylaxis is also recommended for prevention of thromboembolism, should start on admission and should be continued until mobility has been restored. Intermittent pneumatic compression (foot impulse devices) can reduce the risk of VTE. Anti-embolism stockings are effective but are difficult, and sometimes painful, to put on and can cause skin injury in people with fragile skin or vascular insufficiency, or if the stockings are ill-fitting [11]. Nurses should be trained in how to use mechanical prophylaxis and encourage patients to comply.

Other recommended measures to reduce the risk of VTE include the avoidance of dehydration, early surgery, avoidance of prolonged surgery, avoidance of over 
transfusion and early mobilisation [31]. Early mobilisation is simple and particularly effective in lowering the risk of thrombosis, as it increases blood flow, prevents the formation of clots and has an impact on physiological and psychological health with no bleeding complications. Simple exercises, such as walking, repositioning, calf pump exercises and deep breathing help prevent venous stasis [4] and both active and passive leg exercises should be performed to increase blood flow.

\subsubsection{Prevention of Pulmonary Infections}

Early remobilisation is central in the prevention of pneumonia [6,32]. Techniques such as lung expansion manoeuvres (e.g. deep breathing, spirometry) can also reduce the risk of pulmonary complications [14]. Treatment, in addition to antibiotic therapy, includes hydration, a high-calorie/high-protein diet, administration of antipyretics and bronchodilators, rest, oxygen therapy in the case of hypoxemia, monitoring of respiratory status and general health, encouragement of coughing and deep breathing. Patients should be positioned in semi-Fowler position to facilitate breathing, while repositioning helps in loosening lung secretions.

\subsubsection{Prevention of Urinary Tract Infection (UTI)}

UTIs are preventable and early identification of infection leads to prompt treatment and improved outcomes. Early mobilisation is central in prevention and enables patients to maintain self-care and independence while facilitating early removal of catheters. The most important interventions are those of prevention and these are presented in Box 6.4. On suspicion of urinary infection a urine sample should be sent for culture and microbiological analysis followed by appropriate antibiotic therapy. Any indwelling urinary catheter should be removed or replaced before antibiotic administration. Whenever possible, catheters should be avoided or, at least, maintained only for the first $24-48 \mathrm{~h}$ postoperatively. If a catheter is retained for more than $24 \mathrm{~h}$, the reason should be documented and removal should take place as soon as possible, followed by monitoring the patient for retention/incontinence.

\subsubsection{Prevention of Constipation}

The prevention and treatment of constipation involves documentation of stool type (e.g. using the Bristol Stool Scale) and bowel function (e.g. Bowel Function Index), maintaining good nutrition and hydration status, minimising anxiety and maintaining patients' privacy. The overuse of laxatives is a problem which should not be underestimated. The effectiveness of different laxatives does not differ significantly, but the treatment is usually not adjusted satisfactorily to individual needs [33]. Laxatives may not be necessary for patients who are well hydrated and follow a diet rich in fibre (these patients are also more likely to be well nourished) [34]. Prevention and treatment of constipation can also be assisted by good access to toilet facilities, minimising fasting periods and encouraging exercise/mobility. A regular toileting regime (e.g. every $2 \mathrm{~h}$ ) that encourages ambulation and discourages the use of bedpans and an aim for a bowel movement by the second postoperative day (then every $48 \mathrm{~h}$ ) can also assist in prevention [35], but individual patients will differ in what is normal for them. 


\section{Box 6.4: Summary of Evidence-Based Interventions for the Prevention/ Management of the Complications of Stasis}

Deep vein thrombosis

- Risk factors: older age, previous thromboembolism, malignancy, congestive heart failure, obesity, deep venous system disease, surgery, long immobilisation, dehydration

- Assessment: pain, tenderness, changes in skin colour/temperature, oedema (pulmonary embolism: dyspnoea, chest pain, increased respiratory rate, coughing up blood)

- Heparin treatment (low molecular weight/unfractionated)

- Fondaparinux

- Mechanical prophylaxis (intermittent pneumatic compression/foot impulse devices, anti-embolism stockings)

- Avoidance of dehydration

- Early surgery, avoidance of prolonged surgery

- Avoidance of over transfusion

- Early mobilisation

Pulmonary and urinary infections

- Risk factors: older age, poor general health, other infections, cardiopulmonary disease, low albumin, impaired renal function)

- Assessment: cough, sputum production, breaths per minute, type of breathing, saturation levels, dyspnoea, chills, elevated temperature, pleuritic pain, rhonchi/wheezes, use of accessory breathing muscles, cyanosis, mental status

- Techniques such as lung expansion exercises (e.g. deep breathing, spirometry)

- Early ambulation postoperatively

- Hydration, high-calorie/high-protein diet, administration of antipyretics and bronchodilators, rest, oxygen therapy, monitoring, encouragement of coughing/deep breathing

- Semi-Fowler position to facilitate breathing

- Repositioning

Urinary tract infections

- Risk factors: indwelling catheters, older patients, women, dehydration, poor nutrition, diabetes, malignancy

- Assessment: fever, burning during urination/dysuria, urgency and frequency of urination, suprapubic/pelvic pain, haematuria, onset/worsening 
of pre-existing confusion/agitation, urine characteristics (colour, concentration, odour, volume, cloudiness)

- Catheters need not to be put at admission as standard procedure-intermittent catheterisation instead of indwelling catheters

- Analgesic medications administered in the presence of pain

- Good hydration

- Recording of input and output

- Removal of catheters as early as possible postoperatively (after removal, monitoring for retention/incontinence)

- Early identification of infection, prompt treatment

- Preserving functional ability to increase independence and self-care

- Sterile technique for insertion and care:

- Use of lubricant from a single-use container

- Insertion of the smallest lumen catheter possible and instilling $5 \mathrm{~mL}$ in the balloon

- Ensuring that catheter is properly secured (in the abdomen/thigh) to minimise trauma

- Use of closed drainage system

- Sampling port

- Positioning of the drainage system below the level of bladder

- Use of separate clean containers to empty each patient's drainage bag (the bag should not be allowed to fill more than 75\%)

- Encourage/perform routine daily personal hygiene

- Routine catheter care

\section{Constipation}

- Risk factors: pain medications, eating habits, limited activity, lack of privacy

- Assessment: number of bowel movements per week, abdominal distention/ discomfort, abdominal/rectal pain, decreased appetite, nausea, vomiting, bowel obstruction, headache, fatigue, agitation, delirium

- Good nutritional and hydration status (minimum of $1500 \mathrm{~mL}$ of oral fluid daily unless contraindicated) — diet rich in fibre

- Minimisation of extended fasting periods

- Minimisation of anxiety

- Maintaining privacy

- Good accessibility to toilet facilities

- Encouraging exercise and mobility [regular toileting regime (every $2 \mathrm{~h}$ ) that encourages ambulation and discourages the use of bedpans]

- Administration of laxatives 


\subsection{Summary of Key Points}

- Immobility is associated with poor health outcomes and mobilisation and exercise should be encouraged and individualised

- Individual patient goals are determined by pre-fracture mobility/functional status

- Remobilisation is limited by pain, fear and other factors associated with the fall and fracture so mobilisation and pain management should be coordinated

- Rehabilitation should begin as early as possible

- Assessment includes physical function, family/carer involvement, patient beliefs and motivation, education needs and progress towards goals

- Nurses can reduce the risk of falls, assist with mobilisation, ensure walking aids are used and psychologically support patients and carers, but there is insufficient evidence to establish the best strategies for mobility

- Vigilant assessment, prompt interventions and mobilisation prevent the development of complications

- Mechanical prophylaxis along with chemoprophylaxis is recommended for prevention of thromboembolism

- There are simple evidence-based interventions for the prevention of urinary tract infections and constipation.

\subsection{Suggested Further Study}

- Read the following journal papers about the experience of hip fracture:

- Griffiths F et al. (2015) Evaluating recovery following hip fracture: a qualitative interview study of what is important to patients. BMJ Open 5(1):e005406.

- Sims-Gould J, et al. (2017) Patient Perspectives on Engagement in Recovery after Hip Fracture: A Qualitative Study. J Aging Res. Article ID 2171865 https://doi.org/10.1155/2017/2171865

Then write a reflection about what you have learned that relates to mobility and the way in which you and your team practice in relation to helping patients to remobilise. Talk with your colleagues about what you have found and the ways you could use to address possible problems.

- Talk with patients, carers and other staff about the things they feel prevent active remobilisation following surgery. Reflect on what these conversations suggest about how practice might be developed to improve mobility outcomes by involving patients. 


\subsection{Self-Assessment}

To identify learning achieved and the need for further study, the following strategies may be helpful:

- Examine local documentation of nursing care, regarding mobility status and other outcomes and use this to assess knowledge and performance. There is controversy regarding whether the assessment should be of individual nurses or of the nursing team, as, fundamentally, nursing is a team job.

- Seek advice and mentorship from other expert clinicians.

- Meet with specialists and other members of the team to keep up to date on new evidence and disseminate it to colleagues. The conversation in these meetings can include any recent new practices, guidance, knowledge or evidence.

- Review indicators of good practice (e.g. complications incidence, length of stay) and regularly assess patient and carer views and satisfaction. Patient satisfaction has been recognised as an independent indicator of nursing care quality.

- Peer review by colleagues can be used to assess individual progress and practice but should not be too formal. There should be open discussion within the team. Weekly case conferences can identify nurse-focused issues and enable the exchange of expertise. Expertise is conveyed to the various members of the multidisciplinary team by educational initiatives, and by fostering a culture where all the patients' problems are considered.

- There is a lack of published performance indicators and assessment of practical nursing quality, although certain "never events" have been established by nursing associations.

\section{References}

1. Dubljanin-Raspopovic E et al (2012) Use of early indicators in rehabilitation process to predict one-year mortality in elderly hip fracture patients. Hip Int 22:661-667

2. Lee D et al (2014) Prognostic factors predicting early recovery of pre-fracture functional mobility in elderly patients with hip fracture. Ann Rehabil Med 38(6):827-835

3. Salkeld G et al (2000) Quality of life related to fear of falling and hip fracture in older women: a time trade off study. BMJ 320(7231):341-346

4. Nurses Improving Care for Healthsystem Elders (NICHE) (2017) NICHE "Need to knows". http://www.nicheprogram.org/need-to-knows/. Accessed 11 July 2017

5. Maher A et al (2012) Acute nursing care of the older adult with fragility hip fracture: an international perspective (part 1). Int J Orthop Trauma Nurs 16:177-194

6. Handoll $\mathrm{HH}$ et al (2011) Interventions for improving mobility after hip fracture surgery in adults. Cochrane Database Syst Rev (3):CD001704

7. Kamel HK et al (2003) Time to ambulation after hip fracture surgery: relation to hospitalization outcomes. J Gerontol A Biol Sci Med Sci 58(11):1042-1045 
8. Kondo A et al (2010) Determinants of ambulatory ability after hip fracture surgery in Japan and the USA. Nurs Health Sci 12:336-344

9. Abou-Setta AM et al (2011) Comparative effectiveness of pain management interventions for hip fracture: a systematic review. Ann Intern Med 155(4):234-245

10. Egol KA, Strauss EJ (2009) Perioperative considerations in geriatric patients with hip fracture: what is the evidence? J Orthop Trauma 23(6):386-394

11. British Orthopaedic Association (BOA) (2007) The care of patients with fragility fracture. British Orthopaedic Association, London

12. Colón-Emeric CS (2012) Postoperative management of hip fractures: interventions associated with improved outcomes. Bonekey Rep 1:241

13. Copanitsanou P et al (2012) Predictive factors for in-hospital stay and complications after hip fracture. Int J Orthop Trauma Nurs 16:206-213

14. Lo IL et al (2010) Pre-operative pulmonary assessment for patients with hip fracture. Osteoporos Int 21(Suppl 4):S579-S586

15. Roche JJ et al (2005) Effect of comorbidities and postoperative complications on mortality after hip fracture in elderly people: prospective observational cohort study. BMJ 331(7529):1374

16. Halleberg Nyman M et al (2011) A prospective study of nosocomial urinary tract infection in hip fracture patients. J Clin Nurs 20(17-18):2531-2539

17. Maher A et al (2013) Acute nursing care of the older adult with fragility hip fracture: an international perspective (Part 2). Int J Orthop Trauma Nurs 17(1):4-18

18. Johansson I et al (2002) Intermittent versus indwelling catheters for older patients with hip fractures. J Clin Nurs 11(5):651-656

19. Wald $\mathrm{H}$ et al (2005) Extended use of indwelling urinary catheters in postoperative hip fracture patients. Med Care 43(10):1009-1017

20. Polites SF et al (2014) Urinary tract infection in elderly trauma patients: review of the Trauma Quality Improvement Program identifies the population at risk. J Trauma Acute Care Surg 77(6):952-959

21. Kamel HK (2005) The frequency and factors linked to a urinary tract infection coding in patients undergoing hip fracture surgery. J Am Med Dir Assoc 6(5):316-320

22. Trads M, Pedersen PU (2015) Constipation and defecation pattern the first 30 days after hip fracture. Int J Nurs Pract 21(5):598-604

23. Jester R (2007) Evaluating rehabilitation services. In: Jester R (ed) Advancing practice in rehabilitation nursing. Wiley-Blackwell, Oxford

24. Riemen AHK, Hutchison JD (2016) The multidisciplinary management of hip fractures in older patients. Orthop Trauma 30(2):117-122

25. Resnick B et al (2015) Optimizing physical activity among older adults post trauma: overcoming system and patient challenges. Int J Orthop Trauma Nurs 19:194-206

26. Healee DJ et al (2011) Older adult's recovery from hip fracture: a literature review. Int $\mathbf{J}$ Orthop Trauma Nurs 15:18-28

27. Perme C et al (2014) A tool to assess mobility status in critically ill patients: The Perme Intensive Care Unit Mobility Score. Methodist Debakey Cardiovasc J 10(1):41-49

28. Beaupre LA et al (2013) Maximising functional recovery following hip fracture in frail seniors. Best Pract Res Clin Rheumatol 27(6):771-788

29. Sylliaas $\mathrm{H}$ et al (2011) Progressive strength training in older patients after hip fracture: a randomised controlled trial. Age Ageing 40:221-227

30. National Institute for Health and Clinical Excellence (NICE) (2015) Reducing venous thromboembolism risk: orthopaedic surgery. http://pathways.nice.org.uk/pathways/ venous-thromboembolism/reducing-venous-thromboembolism-risk-orthopaedicsurgery\#content=view-node\%3Anodes-hip-fracture

31. Parker M, Johansen A (2006) Hip fracture. BMJ 333(7557):27-30

32. Stolbrink $\mathrm{M}$ et al (2014) The early mobility bundle: a simple enhancement of therapy which may reduce incidence of hospital-acquired pneumonia and length of hospital stay. J Hosp Infect 88(1):34-39 
33. Fosnes GS et al (2011) Effectiveness of laxatives in elderly - a cross sectional study in nursing homes. BMC Geriatr 11:76

34. Sturtzel B et al (2009) Use of fiber instead of laxative treatment in a geriatric hospital to improve the wellbeing of seniors. J Nutr Health Aging 13(2):136-139

35. Mihaylov S et al (2008) Stepped treatment of older adults on laxatives. The STOOL trial. Health Technol Assess 12(13):iii-iiv, ix-139

Open Access This chapter is licensed under the terms of the Creative Commons Attribution 4.0 International License (http://creativecommons.org/licenses/by/4.0/), which permits use, sharing, adaptation, distribution and reproduction in any medium or format, as long as you give appropriate credit to the original author(s) and the source, provide a link to the Creative Commons license and indicate if changes were made.

The images or other third party material in this chapter are included in the chapter's Creative Commons license, unless indicated otherwise in a credit line to the material. If material is not included in the chapter's Creative Commons license and your intended use is not permitted by statutory regulation or exceeds the permitted use, you will need to obtain permission directly from the copyright holder. 